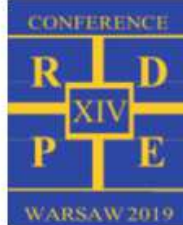

\title{
Influence of unsteady pressure-flow conditions on strength of steel pipelines with volumetric defects reinforced by composite sleeves
}

\author{
Ferdinand Uilhoorn ${ }^{*}$ and Maciej Witek \\ Warsaw University of Technology, Department of Heating and Gas Systems, Nowowiejska 20, 00-653 Warsaw, Poland \\ E-mail addresses: ferdinand.uilhoorn@pw.edu.pl, maciej.witek@pw.edu.pl
}

\begin{abstract}
Structural integrity and risk management have a wide interest because of its practical applications, such as oil and gas pipelines, piping systems under pressure in power stations, urban water, and heating networks. The main goal of this paper is twofold. Firstly, to estimate the unsteady pressureflow variations in a gas transmission grid within the framework of sequential data assimilation. This technique enables to determine accurately the maximum pressure at the localized defect on the pipeline by merging measurements that contain random errors into the inexact numerical flow model. For this purpose, a particle filter is used. The semi-discretization approach is applied to convert the nonisothermal flow model into an initial value problem of ordinary differential equations. The spatial discretization is based on a five-point, fourth-order finite difference approximation and the time marching was done using a diagonally implicit Runge-Kutta scheme. Secondly, to study the strength of steel tubes reinforced with composite sleeves containing localized part-wall thickness losses caused by corrosion while taking into consideration a safe operating pressure. For a steel thin-walled cylinder containing a wrap of fiberglass with epoxy resin, the burst pressure and sleeve thickness are determined. Finally, the repaired pipeline with a fiber-reinforced composite sleeve is investigated. The results enable operators to handle problems of corroded steel pipelines and develop effective repair activities during operation. For this reason, current research is important for the maintenance of underground steel networks.
\end{abstract}

\section{Introduction}

Magnetic flux leakage (MFL) technology using axial excitation of magnetic field lines is the most effective and widespread in-line inspection method for longdistance high-pressure underground pipelines [1]. The data gives insight into tube wall losses along the pipeline. Defect assessment after periodic diagnostics plays an important role for safe operation of natural gas grids [2]. It enables to evaluate the severity of flaws and to select the anomalies to be repaired with polymer sleeves. A three-step methodology was employed in [3] as a useful tool for operators to evaluate the severity of part-wall metal losses and aging process of underground steel pipelines that transport natural gas. Time-dependent reliability of corroded steel pipelines with correlations of random variables was presented inter alia in [4].

The role of pressure is utmost important in estimating the tube structural integrity. In previous work [5] a steady-state flow model was used to determine the internal pressure as a main load of the pipe wall. This steady-state approach is adequate if the system is not subject to significant changes in flow and pressure. In this research, a more realistic situation is investigated, namely unsteady flow conditions. Moreover, these transients are considered as realizations of a stochastic process. As far as the authors are aware, such an approach has a poor representation in the literature.
Flow models are inexact and contain uncertainties that are related to initial and boundary conditions, fluctuations in gas composition, ambient temperature, soil properties and so on. As a result, the estimates of state variables such as pressure, temperature and flow rate might too much deviate from the reality. To handle this problem, an ensemble data assimilation approach is implemented. In particular, the particle filter $[6,7,8]$ which provides a framework for solving estimation problems that is not limited to Gaussianity and has attractive convergence properties.

The aim of this study is to investigate the strength of pipelines containing volumetric defects due to general and pitting corrosion observed by in-line inspection. The decrease in the tube wall thickness caused by electrochemical corrosion is analysed considering the limit state of plastic collapse and corresponding maximum operating pressure of the remaining pipe wall residual thickness. This value was compared to the estimated internal pressure obtained from the data assimilation approach at the point of the localized defect. Thickness of multi-layer fiber-reinforced composite sleeve was determined in order to reach the minimum strength of the pipeline.

Structural integrity and risk management have a wide interest because of its practical applications, such as oil and gas steel pipelines [5,9], piping systems under pressure in power stations, urban water and heating 
networks. This research enables network operators of steel pipelines subjected to corrosion to handle capacity limitations based on transient pressure calculations.

\section{Nonisothermal gas flow model}

The compressible gas flow model constitutes a nonhomogeneous hyperbolic system of partial differential equations (PDEs) derived from the conservation principles of mass, momentum and energy. Using a primitive formulation, the governing equations can be written as [10].

$$
\frac{\partial \mathbf{W}}{\partial t}+\mathbf{F}(\mathbf{W}) \frac{\partial \mathbf{W}}{\partial x}+\mathbf{S}(\mathbf{W})=0
$$

with $\forall x \in \mathbb{R}, t \in \mathbb{R}^{+}, \mathbf{W}=\left[\begin{array}{lll}p & \dot{m} & T\end{array}\right]^{\top}$ and

$$
\begin{aligned}
& \mathbf{F}(\mathbf{W})=\left[\begin{array}{c}
-\frac{\dot{m}\left(a_{s}^{2} \alpha_{2}-R T z\right)}{A p} \\
A-\frac{a_{s}^{2} \alpha_{2}^{2} c_{p} \dot{m}^{2}-R a_{s}^{2} \alpha_{1}^{2} \alpha_{2} \dot{m}^{2} z}{A c_{p} p^{2}} \\
-\frac{R T a_{s}^{2} \alpha_{1} \alpha_{2} \dot{m} z}{A c_{p} p^{2}}
\end{array}\right. \\
& \frac{a_{s}^{2}}{A} \\
& \frac{\dot{m}\left(\alpha_{2} c_{p} a_{s}^{2}-R z a_{s}^{2} \alpha_{1}^{2}+R T c_{p} z\right)}{A c_{p} p} \\
& \frac{R T a_{s}^{2} \alpha_{1} z}{A c_{p} p}
\end{aligned}
$$



$$
\begin{aligned}
& \frac{R \dot{m} z\left(a_{s}^{2} \alpha_{1}^{2}+T c_{p}\right)}{A c_{p} p} \\
& \mathbf{S}(\mathbf{W})=\left[\begin{array}{c}
-\frac{a_{s}^{2} \alpha_{1}(\Omega A p+R T \dot{m} w z)}{A^{2} T c_{p} p} \\
w+\frac{p A g}{z T R} \sin \theta \\
-\frac{a_{s}^{2} \alpha_{2}(\Omega A p+R T \dot{m} w z)}{A^{2} c_{p} p^{2}}
\end{array}\right],
\end{aligned}
$$

with pressure $p$, mass flow rate $\dot{m}$, temperature $T$, crosssectional area $A$, frictional force $w$, gravitational acceleration $g$, angle of inclination $\theta$, rate of heat transfer $\Omega$, specific gas constant $R$, compressibility factor $z$ and specific heat at constant pressure $c_{p}$. The parameters $\alpha_{l}$ and $\alpha_{2}$ are calculated as follows

$$
\alpha_{1}=1+\frac{T}{z}\left(\frac{\partial z}{\partial T}\right)_{p}, \quad \alpha_{2}=1-\frac{p}{z}\left(\frac{\partial z}{\partial p}\right)_{T} .
$$

The isentropic wave speed $(\partial p / \partial \rho)_{s}^{1 / 2}$ is denoted as $a_{s}$ and obtained from the expression

$$
\begin{aligned}
\left(\frac{\partial p}{\partial \rho}\right)_{s} & =\left[\frac { \rho } { p } \left(1-\frac{p}{z}\left(\frac{\partial z}{\partial p}\right)_{T}\right.\right. \\
& \left.-\frac{p}{\rho c_{p} T}\left(1+\frac{T}{z}\left(\frac{\partial z}{\partial T}\right)_{p}\right)^{2}\right]^{-1},
\end{aligned}
$$

where $\rho$ is the density. The frictional force per unit length is defined as

$$
w=\frac{1}{8} f_{r} \rho v|v| \pi D_{i},
$$

with velocity $v$ and internal diameter $D_{i}$. The friction factor $f_{r}$ is obtained from the Colebrook-White equation [11]

$$
\frac{1}{\sqrt{f_{r}}}=-2 \log \left(\frac{\varepsilon}{3.7 D_{i}}+\frac{2.51}{\operatorname{Re} \sqrt{f_{r}}}\right),
$$

where $\varepsilon$ denotes the roughness and Re refers to Reynolds number. The steady heat transfer between the fluid and soil per unit length and time is defined as

$$
\Omega=-\pi d U\left(T-T_{s}\right),
$$

where $U$ is the total heat transfer coefficient and $T_{s}$ the soil temperature. Finally, to the flow model, the following initial and boundary conditions are imposed:

$$
\begin{gathered}
p(x, 0)=p_{0}(x), \quad T(x, 0)=T_{0}(x), \\
\dot{m}(x, 0)=\text { const. } \\
p(0, t)=p_{0}, \quad \dot{m}(\ell, t)=\phi(t), \quad T(0, t)=T_{0},
\end{gathered}
$$

respectively. The pipeline length is denoted as $\ell$.

The numerical approximation is done using the semidiscretization approach, which converts the flow model into ordinary differential equations (ODEs) via discretization of the spatial derivatives. The sparse system of ODEs reads

$$
\frac{\mathrm{d} \mathbf{w}(t)}{\mathrm{d} t}=-\mathbf{F}(\mathbf{w}) \mathbf{T w}(t)-\mathbf{S}(\mathbf{w}(t))=f(\mathbf{w}(t)),
$$

with $\mathbf{w}\left(t_{0}\right)=\mathbf{w}_{0}$ and

$$
\begin{gathered}
\mathbf{w}(t)=\left[p_{1}(t), \cdots, p_{i}(t), \cdots, p_{n_{x}}(t), \dot{m}_{1}(t), \cdots,\right. \\
\left.\dot{m}_{i}(t), \cdots, \dot{m}_{n_{x}}(t), T_{1}(t), \cdots, T_{i}(t), \cdots, T_{n_{x}}(t)\right]^{\top},
\end{gathered}
$$

whereas the discretization is done uniformly on the interval $[0, \ell]$ with $n_{x}$ nodes. In Eq. (11), the parameter $\mathbf{T}$ represents a block-diagonal matrix defined as $\mathbf{T}=\sum_{j=1}^{n} \mathbf{I}_{j} \otimes \mathbf{T}^{(j)}$. Since $n=3$ and the sub-matrices are identical, $\mathbf{T}^{(j)}=\mathbf{T}^{(1)} \forall j$ where 


$$
\mathbf{T}^{(1)}=\frac{1}{2 \Delta x}\left[\begin{array}{cccccc}
-3 & 4 & -1 & 0 & \cdots & 0 \\
-1 & 0 & 1 & 0 & \cdots & 0 \\
0 & -1 & 0 & 1 & \cdots & 0 \\
\vdots & \ddots & \ddots & \ddots & \ddots & \vdots \\
0 & \cdots & 0 & -1 & 0 & 1 \\
0 & \cdots & 0 & 1 & -4 & 3
\end{array}\right] .
$$

The coefficients in the sub-matrix refer to the classical three-point finite difference approximation.

For the time stepping, a second order diagonally implicit Runge-Kutta (DIRK) method [12,13] is used. This scheme starts with the trapezoidal rule (TR) followed by the second order backward-differentiation formula (BDF2) and showed to be robust with good accuracy and efficiency properties, if the tolerance is not set too tight [14]. Within the framework of particle filtering, the large set of ODEs is sampled many times, therefore in order to reduce the computation time, the sparsity of the system is exploited.

\section{Particle filter}

The idea of data assimilation is to combine noisy measurements with the numerical flow model. The heart of the approach is based on Bayes theorem that states that the model pdf can be updated via measurements. Mathematically, it reads:

$$
\begin{gathered}
\zeta_{k}=g\left(\zeta_{k-1}\right)+v_{k-1}, \\
y_{k}=\zeta_{k}+n_{k},
\end{gathered}
$$

where $\zeta_{k} \in \mathbb{R}^{n_{\zeta}}$ refers to the states, $y_{k} \in \mathbb{R}^{n_{y}}$ denote the measurements, $\quad v_{k} \in \mathbb{R}^{n_{v}}$ and $n_{k} \in \mathbb{R}^{n_{n}}$ are i.i.d. random variables that represent the model and measurement noise, respectively. The noise statistics are not unimportant, if wrongly selected filter divergence might occur. On the other hand, a priori knowledge about these statistics is often based on trial-and-error or tuning [15]. Here $g: \mathbb{R}^{n_{\zeta}} \times \mathbb{R}^{n_{v}} \mapsto \mathbb{R}^{n_{\zeta}}$ refers to the finite dimensional model. In Eq. (15) it can be seen that the state variables of interest, namely, pressure, temperature and mass flow rate are directly measured. However, the state variables contain noise that we seek to reduce by data assimilation. A technique that is used here is particle filtering where the aim is to approximate the posterior pdf $\pi\left(\zeta_{0: k} \mid y_{1: k}\right)$ where $\zeta_{0: k}=\left\{\zeta_{0}, \zeta_{1}, \ldots, \zeta_{k}\right\} \quad$ and $y_{1: k}=\left\{y_{1}, y_{2}, \ldots, y_{k}\right\}$. The advantage of particle filters is that they are not limited by linearity or Gaussianity. This technique is tracking a set of states $\zeta_{0: k}$ up to time $k$ by updating a random measure $\left\{\zeta_{0: k}^{i}, \omega_{k}^{i}\right\}_{i=1}^{N_{p}}$ that $\quad$ approximates $\pi\left(\zeta_{0: k} \mid y_{1: k}\right)$. Here $\left\{\zeta_{0: k}^{i}, i=0, \ldots, N_{p}\right\}$ is a set of support points and associated weights $\left\{\omega_{k}^{i}, i=1, \ldots, N_{p}\right\}$. The particle filter starts with sampling $N_{p}$ particles $\zeta_{0}^{i}$ from the initial model probability density $\pi\left(\zeta_{0}\right)$ followed by integrating all particles in time up to the moment measurements become available, i.e., $\zeta_{k}^{i} \sim \pi\left(\zeta_{k} \mid \zeta_{k-1}^{i}\right) \forall i$. In this work, the importance density $\pi\left(\zeta_{k} \mid \zeta_{k-1}^{i}, y_{k}\right)$ is set equal to the prior $\pi\left(\zeta_{k} \mid \zeta_{k-1}^{i}\right)$. The next step is to compute the weights $\omega_{k}^{i}=\pi\left(y_{k} \mid \zeta_{k}^{i}\right) / \sum_{i=1}^{N_{p}} \pi\left(y_{k} \mid \zeta_{k}^{i}\right) \quad$ followed by resampling of particles such that the weights are equal to $N_{p}^{-1}$. This sequence is repeated for all measurements. In the resampling step particles with low weights are excluded, whereas particles with high weight are kept for the posterior pdf. The reader is referred to $[6,7,8]$ for details.

It is known, that particle filters might suffer from the curse of dimensionality, this means that the ensemble size to generate a few samples from the high-probability areas of the posterior is growing exponentially with the dimension of the state vector $[16,17]$. However, in the numerical experiments a coarse interval is used, and the sampling is done only at the inlet and outlet node of the spatial discretization. For this reason, it is reasonable to assume that the curse of dimensionality does not apply here.

\section{Strength of defected tube}

The Det Norske Veritas Recommended Practice methodology [18] was selected as it is considered adequate for electrochemical corrosion defects and steel grade investigated in this work. The maximum allowable operating pressure of the defected pipeline with longitudinal oriented single metal-loss (see Fig. 1) is defined by [18]

$$
M A O P_{\mathrm{DNV}}=\gamma_{m} \frac{2 \delta f_{u}\left(1-\gamma_{d}\left(\frac{d}{\delta}\right)^{*}\right)}{\left(D_{o}-\delta\right)\left(1-\frac{\gamma_{d}\left(\frac{d}{\delta}\right)^{*}}{Q}\right)},
$$

where

$$
\begin{aligned}
\left(\frac{d}{\delta}\right)^{*} & =\left(\frac{d}{\delta}\right)_{\text {means }}+\varepsilon_{d} S t D\left(\frac{d}{\delta}\right), \\
Q & =\sqrt{1+0.31\left(\frac{L^{2}}{D_{o} \delta}\right)},
\end{aligned}
$$

with outside diameter $D_{\mathrm{o}}$, ultimate tensile strength of the pipe $f_{\mathrm{u}}$ to be used in the design, single defect depth $d$ derived from diagnostics, defect length $L$ derived from 
in-line inspection, wall nominal thickness $\delta$, measured (relative) single defect depth $(d / \delta)_{\text {means }}$ obtained from the inspection results, and standard deviation of the measured ratio $S t D(d / \delta)$ derived from the specification of MFL tool by the assumption of normal distribution of random variable $d / \delta[18]$.

The safety factors $\gamma_{\mathrm{m}}, \gamma_{\mathrm{d}}$ and $\varepsilon_{\mathrm{d}}$ in Eq. (16) and (17) are determined by: (i) pipeline safety class, usually derived from design, (ii) testing method, relative or absolute and (iii) inspection accuracy and a confidence level. According to the Polish regulation, the degree of area urbanization concerning the location of highpressure gas pipelines is classified as follows:

- Class 1 (safety class high corresponding to the pipeline segment design factor $f \leq 0.4$ ) covers an area built over with single- or multi-family collective dwelling houses and public utility facilities, with intensive vehicular traffic and developed underground infrastructure, such as water networks, sewage systems, heat distribution networks, gas grids, power and telecommunication networks, as well as streets, roads and mining sites;

- Class 2 (safety class normal corresponding to the pipeline segment design factor $0.4<f \leq 0.6)$ comprises an area with single-family and farmstead housing and individual leisure buildings, along with their necessary infrastructure;

- Class 3 (safety class low corresponding to the pipeline segment design factor $0.6<f \leq 0.72$ ) includes an undeveloped area and an area in which only isolated single-family houses and farm and livestock buildings can be situated, along with their necessary infrastructure.

The safety factor $\gamma_{\mathrm{m}}$ is obtained from in-line inspections that are based on relative feature depth measurements such as the MFL method. Its value equals $\gamma_{\mathrm{m}}=0.79$ for class location low. The partial safety factor for corrosion depth is $\gamma_{\mathrm{d}}=1.156$. The fractile value for corrosion $\varepsilon_{\mathrm{d}}=0$. The latter two factors depend on the safety class and standard deviation and selected according to [18].
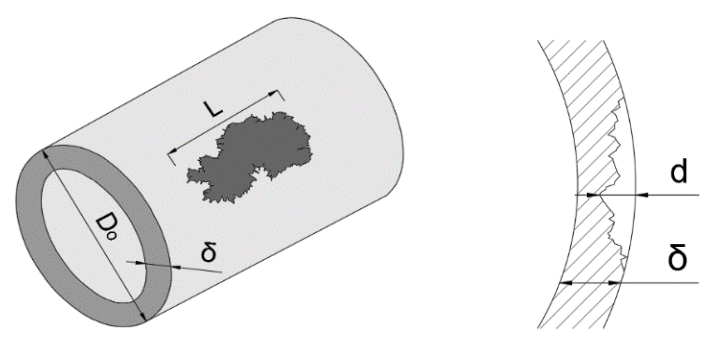

Fig. 1. Geometry of the axially oriented tube wall material loss.

\section{Pipe strength repaired by composite sleeve}

The components of a multi-layer polymer repair system applied to a steel pipe with volumetric defect in current research as well as in publications $[3,20]$ is shown in Fig. 2.

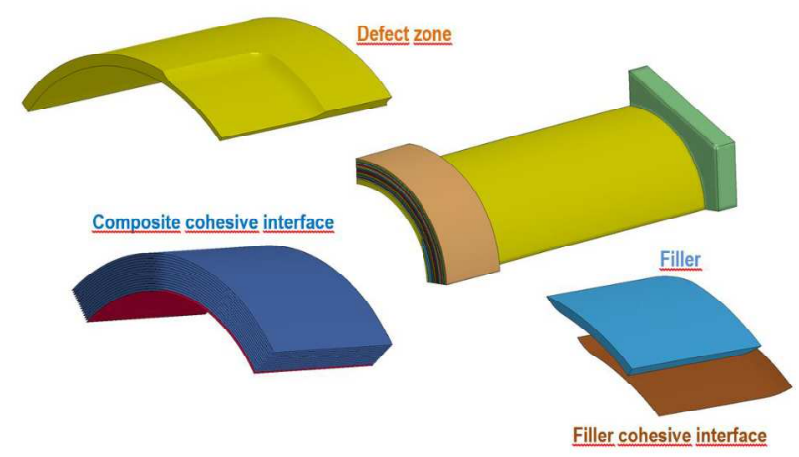

Fig. 2. Layered system of composite repair used for defected steel pipe reinforcement.

The strength calculations applied in this paper are reasonable for estimating the failure pressure of a corroded pipe, reinforced with a multi-layer composite wrap [19]. Assuming an elastic behavior of both steel tube and polymer sleeve, the failure pressure of a straight, thin-walled cylinder with volumetric defect reinforced by composite wrap can be calculated as follows $[3,19]$

$$
P_{\max }^{\mathrm{th}}=\frac{f_{u}\left(r_{o}-r_{i}\right)}{\alpha_{\theta}\left(r_{i}-\eta r_{o}\right)}
$$

where

$$
\begin{gathered}
\alpha_{\theta}=\left[\frac{1-\left(\frac{d}{\delta}\right)\left(\frac{1}{Q}\right)}{1-\left(\frac{d}{\delta}\right)}\right], \\
\eta=\left(\frac{r_{o}^{2} E_{\text {pipe }}\left(r_{o}-r_{i}\right)}{r_{i}^{2} E_{\text {sleeve }}\left(r_{e}-r_{o}\right)}+\frac{r_{o}}{r_{i}}\right)^{-1},
\end{gathered}
$$

with failure pressure of a straight pipe with volumetric defect reinforced by composite wrap $P_{\max }^{\text {th }}$, external radius of the steel tube plus thickness of the sleeve $r_{\mathrm{e}}$, inner radius of the steel pipe $r_{i}$, external radius of the steel pipe $r_{0}$, elasticity modulus of steel $E_{\text {pipe }}$ and elasticity modulus of the composite sleeve $E_{\text {sleeve }}$.

The elasticity modulus of a sleeve depends on the polymer and other components of the layered system used for the pipeline reinforcement. However, elasticity modulus of the sleeve in fiber-reinforced composite wraps depends on the fiber volume. According to [3], the elasticity modules of the fiberglass sleeve with $50 \%$ of fiber equals to $44.2 \mathrm{GPa}$. Analyses of fiberglass reinforcement systems such as applied in [20] confirm the assumption that the resin volume in a sleeve is 
negligible and an averaged elasticity modulus of the polymer in the hoop direction is approximately equal to a mean of an axial and a transverse value. These calculation assumptions were applied in [3]. The elasticity moduli used in this work are shown in Table 1.

Table 1. Elasticity modulus of steel, fiberglass sleeve and epoxy filler [3].

\begin{tabular}{|c|c|c|c|c|}
\hline $\begin{array}{c}\text { Elasticity } \\
\text { modulus } \\
\text { of steel } \\
\mathrm{E}_{\text {pipe }} / \mathrm{GPa}\end{array}$ & $\begin{array}{c}\text { Axial } \\
\text { modulus of } \\
\text { fiberglass } \\
\text { sleeve } \\
\mathrm{E}_{\mathrm{Gx}} / \mathrm{GPa}\end{array}$ & $\begin{array}{c}\text { Transverse } \\
\text { modulus of } \\
\text { fiberglass } \\
\text { sleeve } \\
E_{\mathrm{Gy}} / \mathrm{GPa}\end{array}$ & $\begin{array}{c}\text { Axial } \\
\text { modulus } \\
\text { of epoxy } \\
\text { resin } \\
E_{\mathrm{Rx}} / \mathrm{GPa}\end{array}$ & $\begin{array}{c}\text { Transverse } \\
\text { modulus of } \\
\text { epoxy resin } \\
E_{R y} / \mathrm{GPa}\end{array}$ \\
\hline 206 & 44.2 & 44.2 & 3.4 & 3.4 \\
\hline
\end{tabular}

The acceptability condition of tube wall metal loss without reinforcement can be written as follows

$$
P(x)_{\mathrm{est}} \leq M A O P_{\mathrm{DNV}},
$$

with estimated pressure from the flow model $P(x)_{\text {est }}$ at the metal loss coordinate $\mathrm{x}$ along the pipeline, maximum allowable operating pressure of the defected thin-walled cylinder with longitudinal oriented single metal-loss $M A O P_{\mathrm{DNV}}$ calculated according to [18].

The thickness of the multi-layer composite sleeve $\left(k=r_{\mathrm{e}}-r_{\mathrm{o}}\right.$ ) has to be determined in order to reach the minimum strength of the reinforced tube. This to resist the estimated operating pressure at the point of the defect occurrence, i.e.,

$$
P(x)_{\mathrm{est}} \leq f P_{\max }^{\mathrm{th}},
$$

with design factor $f$ depending on the pipeline segment safety class.

The condition for maximum admissible operating pressure of the pipeline with volumetric surface flaw reinforced with composite sleeve can be expressed by the inequality:

$$
M A O P^{\text {th }} \leq f P_{\max }^{\text {th }}
$$

with maximum admissible operating pressure of the thinwalled cylinder containing volumetric surface defect reinforced with multi-layer composite sleeve $M A O P^{\text {th }}$.

If as usual in steel underground structures maintenance, many pipe wall metal losses were recognized as a result of in-line inspection, the conditions (23) and (24) have to be fulfilled for each volumetric surface defect.

\section{Case study}

In the first part of the case study, the flow dynamics are estimated using the particle filter. From the spatial-temporal evolution of the pressure estimates, the maximum pressure is determined at the defect location. This point is obtained from hypothetical MFL diagnostics. In the second part, the maximum allowable operating pressure of the tube with part-wall metal loss is computed and compared with the estimated internal fluid pressure obtained from the flow model. Based on these calculations the strength of the defected pipe, reinforced with fiber-based polymer sleeve is analyzed in order to determine the appropriate thickness of multi-layered repair wrap.

\subsection{Pressure estimates}

For the investigation, a section of the Yamal transit pipeline on Polish territory between two compressor stations was selected with the following parameters: $\ell=$ $177 \mathrm{~km}, D_{\mathrm{i}}=1.380 \mathrm{~m}, \varepsilon=0.0015 \mathrm{~mm}, T_{s}=12^{\circ} \mathrm{C}$ and $U$ $=1.66 \mathrm{~W} \mathrm{~m}^{-2} \mathrm{~K}^{-1}$. The molar fraction of the natural gas is: $98.3455 \mathrm{CH}_{4}, 0.6104 \mathrm{C}_{2} \mathrm{H}_{6}, 0.1572 \mathrm{C}_{3} \mathrm{H}_{8}, 0.0299 i$ $\mathrm{C}_{4} \mathrm{H}_{10}, 0.0253 n-\mathrm{C}_{4} \mathrm{H}_{10}, 0.0055 i-\mathrm{C}_{5} \mathrm{H}_{12}, 0.0040 n-\mathrm{C}_{5} \mathrm{H}_{12}$, $0.0303 \mathrm{~N}_{2}$ and $0.7918 \mathrm{CO}_{2}$. The thermodynamic and transport properties required in flow model (1) were fitted to the calculated values of GERG-2008. This equation of state is highly accurate, but computationally intensive and therefore, less practically simulating flow transients. Therefore, the following multivariate regression model is used:

$$
\kappa(p, T)=\sum_{i=1}^{n} \iota_{i} p^{\mathcal{I}_{i, 1}} T^{\mathcal{I}_{i, 2}},
$$

with $\mathcal{I}$ as the power and $\iota_{i}$ refer to the corresponding coefficients and 5 degrees freedom. The simulations were done on the interval $t_{\mathrm{f}} / \mathrm{h} \in[0,24]$ and with $p(0, t)=$ $8.4 \mathrm{MPa}, T(0, t)=308.15 \mathrm{~K}$ and $\dot{m}(\ell, t)=f(t)$. The latter boundary condition is depicted in Fig. 3 .

In the absence of real data for the state variables, it is common to generate synthetic data and to avoid inverse crime, the numerical model is solved on a coarser grid $\left(n_{x}=101\right)$ than the one that is used to generate the data $\left(n_{x}=1001\right)$. The measurements were generated by adding a random Gaussian noise to the model solutions with a fine grid, i.e., $n_{k, p} \sim \mathcal{N}\left(0,0.04^{2}\right), \quad n_{k, T} \sim \mathcal{N}\left(0,1.5^{2}\right) \quad$ and $n_{k, \dot{m}} \sim \mathcal{N}\left(0,2.5^{2}\right)$ with variance in $\mathrm{MPa}^{2}, \mathrm{~K}^{2}$ and $(\mathrm{kg}$ $\left.\mathrm{s}^{-1}\right)^{2}$, respectively. It is assumed that the model noise is based on Gamma random variables, i.e., $v_{k-1, p} \sim \operatorname{Gamma}(9,0.5), \quad v_{k-1, T} \sim \operatorname{Gamma}(5,1)$ and $v_{k-1, \dot{m}} \sim \operatorname{Gamma}(3,2)$. The Gaussian and Gamma random variables were generated using Matlab functions randn and gamrnd. The ensemble size $N_{p}$ is set to 100 . The measurement sampling time is set to $10 \mathrm{~min}$ but in practice, it can vary from one to several tens of minutes. The spatial-temporal evolution of the filtered estimates for all state variables is shown in Fig. 4.

It is assumed that the part-wall volumetric defect is located at $60 \mathrm{~km}$ from the up-stream compressor station. Since, the distance does not coincide with a grid point after spatial discretization, the pressure values are obtained from its nearest values using piecewise cubic interpolation. The maximum pressure at $x=60 \mathrm{~km}$ is $P_{\text {est }}$ $(60)=8.37 \mathrm{MPa}$ after $16 \mathrm{~h}$ from the beginning of the simulation (Fig. 5). 


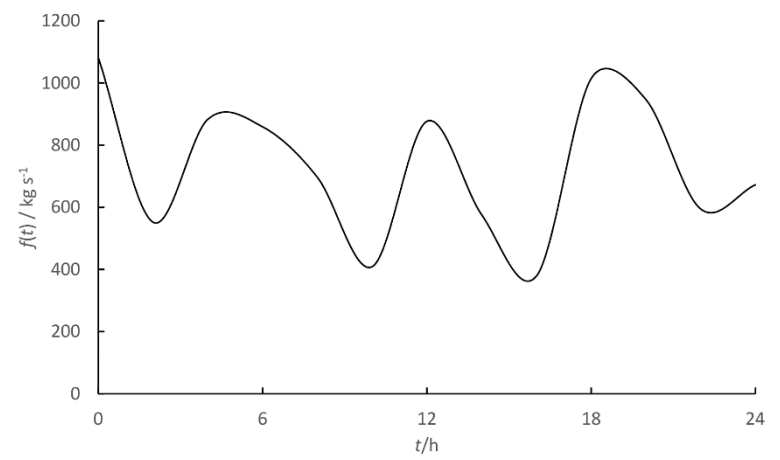

Fig. 3. Mass flow rate boundary condition.

\subsection{Strength of steel tube repair}

In the second part of this case study, the allowable pressure is calculated based on the following pipeline parameters [21]: $f_{\mathrm{u}}=570.0 \mathrm{MPa}, f_{\mathrm{y}}=485 \mathrm{MPa}, E_{\text {pipe }}=$ $206 \mathrm{GPa}, D_{o}=1422 \mathrm{~mm}, \delta=21 \mathrm{~mm}, r_{\mathrm{o}}=711 \mathrm{~mm}$ and $r_{\mathrm{i}}=690 \mathrm{~mm}$. Dimensions of the longitudinal oriented corrosion area shown in Fig. 1 are derived from hypothetical in-line inspection results. The maximum defect depth as a percentage of nominal tube wall thickness is $(d / \delta)_{\text {means }}=43 \%$ and the axial length $L$ of the corroded area is $1000 \mathrm{~mm}$.

Based on the feature dimensions obtained from hypothetical MFL diagnostics, the maximum allowable operating pressure of the defected pipe without reinforcement calculated from the formula (16) equals to $M A O P_{\mathrm{DNV}}=7.98 \mathrm{MPa}$. The maximum pressure obtained from the data assimilation approach at the defect location equals to $P_{\text {est }}(60)=8.37 \mathrm{MPa}$. This means that condition (22) is not fulfilled and therefore the pipeline must be immediately excavated, and the external surface flaw needs to be directly assessed as it was described in [2]. If the inspection tool defect sizing is confirmed, the volumetric metal loss feature has to be reinforced by composite wrap in order to reach minimum required strength of the tube. Determination results of multi-layer polymer sleeve thickness $k$ to reach the strength of the defected pipe corresponding to the maximum pressure at the point of the specific defect occurrence is presented below. For the number of layers of the composite wrap $k$ $=16$, the corresponding burst pressure of the repaired tube equals to $P_{\max }^{\text {th }}=11.63 \mathrm{MPa}$. From Eq. (24), the maximum admissible operating pressure while considering a low safety class for the pipeline that contains a volumetric surface defect reinforced with a composite sleeve equals to $M A O P^{\text {th }}=8.37 \mathrm{MPa}$. Hence, for the specific defect dimensions and $k=16$ obtained from Eqs (19-21), condition (23) is satisfied.
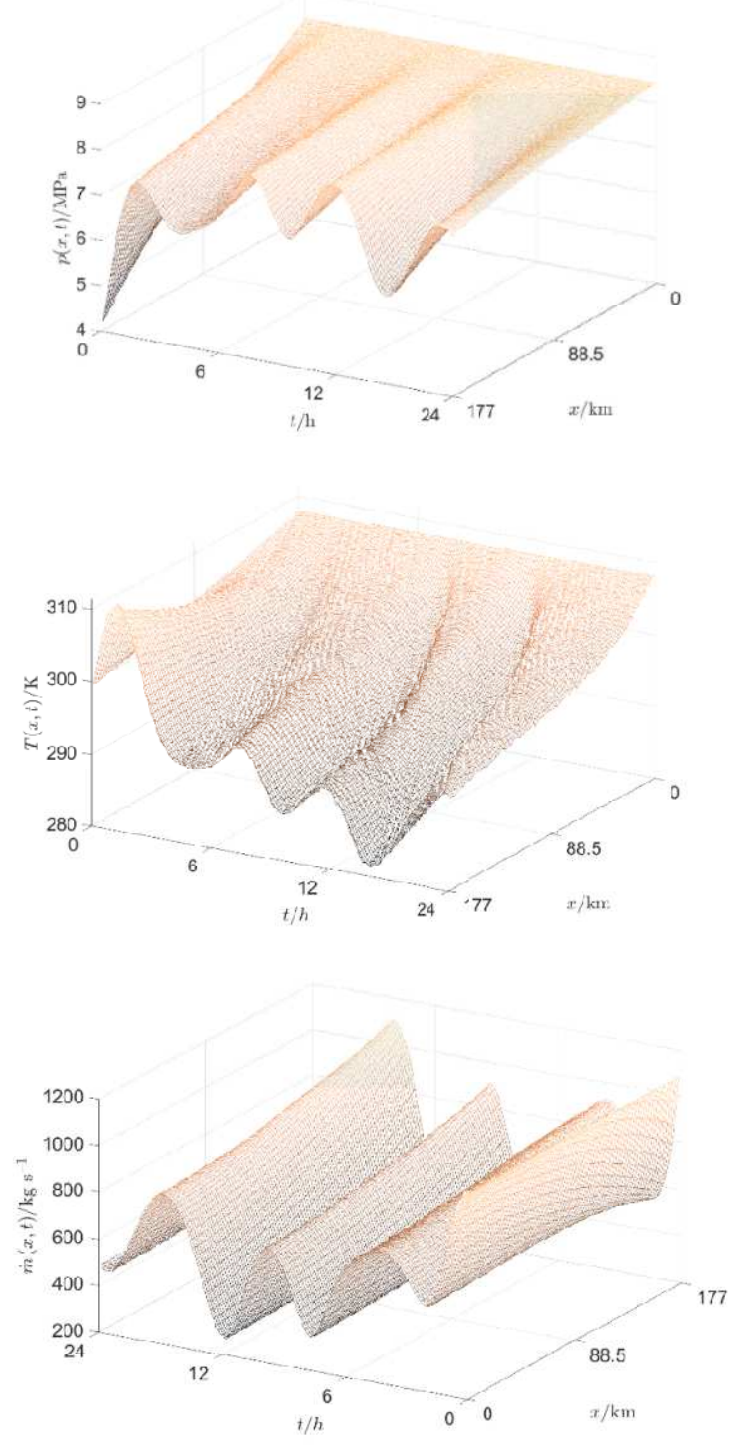

Fig. 4. Evolution of state variables.

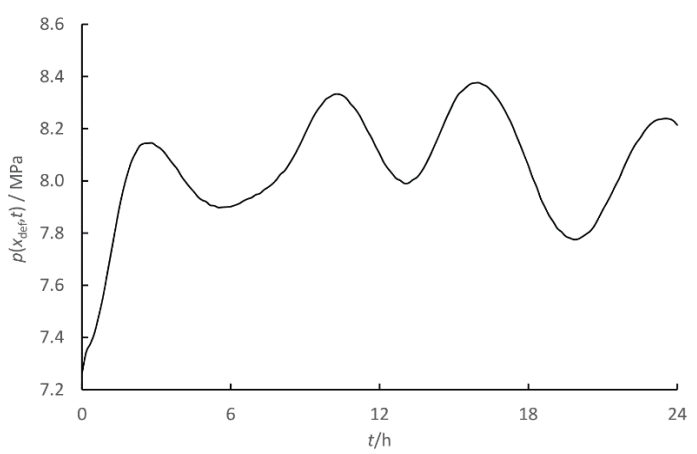

Fig. 5. Pressure conditions at the volumetric defect location. 


\section{Conclusions}

In this work, a data assimilation technique was used to estimate the pressure under transient flow conditions at the point where cylinder wall defect exists in order to evaluate the underground pipeline integrity. This approach is more accurate and realistic compared to a situation where the pressure at the certain coordinate does not change in time, which is nowadays assumed in thin-walled tubes strength analysis.

Repairs of part-wall surface metal losses derived from periodic in-line inspection by composite sleeves avoid unnecessary capacity limitations of steel network subjected to corrosion due to local reinforcement of pipe in order to maintain design-operating pressure of the infrastructure. The analytical strength computations applied in the current paper is reasonable to estimate the failure pressure of corroded tubes reinforced with polymer sleeves. A more accurate strength analysis requires a numerical model, which enables to simulate a multi-layered repair system. This approach is more complicated but recommended for further research.

\section{References}

1. Bouledroua O., Zelmati D., Hassai M., Inspections, statistical and reliability assessment study of corroded pipeline, Engineering Failure Analysis Vol. 100, pp. 1-10, (2019).

2. Witek M., Validation of in-line inspection data quality and impact on steel pipeline diagnostic intervals, Journal of Natural Gas Science and Engineering, Vol. pp. 121-133, (2018).

3. Witek M., Gas transmission failure probability estimation and defect repairs activities based on inline inspection data, Engineering Failure Analysis, Vol. 70, pp. 255-272, (2016).

4. Zhou W., System reliability of corroding pipelines, International Journal of Pressure Vessels and Piping, Vol. 87, pp. 587-595, (2010).

5. Witek M., Batura A., Orynyak I., Borodii M., An integrated risk assessment of onshore gas transmission pipelines based on defect population, Engineering Structures, Vol. 173, pp. 150-165, (2018).

6. Arulampam S., Maskell S., Gordon N., Clapp T., A tutorial on particle filters for on-line non-linear/nonGaussian Bayesian tracking, Signal Processing, IEEE Transactions on Vol. 50 (2), pp. 174, 2002.

7. Doucet A., On sequential Monte Carlo methods for Bayesian filtering. Tech. rep., Tech. Rep., Univ. Cambridge, Department of Engineering, UK, (1998).

8. Gordon N., Salmond D., Smith A., Novel approach to nonlinear/non-Gaussian Bayesian state estimation, Radar and Signal Processing, IEE Proceedings F Vol. 140(2), pp. 107-113, (1993).

9. Han Z.Y., Weng W.G., An integrated quantitative risk analysis method for natural gas pipeline network, Journal of Loss Prevention in the Process Industries, Vol. 23, pp. 428-436, (2010).
10. Uilhoorn F.E., Comparison of Bayesian estimation methods for modeling flow transients in gas pipelines, Journal of Natural Gas Science and Engineering, Vol. 38, pp. 159-170, (2017).

11. Colebrook C., Turbulent flow in pipes, with particular reference to the transition region between the smooth and rough pipe laws, J. Inst. Civil Eng. Vol. 11, pp. 133-156, (1939).

12. Bank R.E., Coughran, Jr. W.M., Fichtner W., Grosse E. H., Rose D. J., Smith R. K., Transient Simulation of Silicon Devices and Circuits, IEEE Transactions on Computer-Aided Design CAD, Vol 4(4), pp. 436-451 (1985).

13. Shampine L., Hosea M., Analysis and implementation of TR-BDF2, Applied Numerical Mathematics, Vol. 20(1-2), pp. 21-37, (1996).

14. Uilhoorn F.E., A comparison of numerical integration schemes for particle filter-based estimation of gas flow dynamics, Physica Scripta, Vol. 93 (12), (2018).

15. Uilhoorn F.E., A multiobjective optimization approach to filter tuning applied to coupled hyperbolic PDEs describing gas flow dynamics, Optimal Control Applications and Methods, Vol. 40 (4), pp. 750-763, (2019).

16. Bickel P., Li B., Bengtsson T., Sharp failure rates for the bootstrap particle filter in high dimensions, IMS Collections: Pushing the Limits of Contemporary Statistics: Contributions, pp. 318329, (2018).

17. Snyder C., Bengtsson T., Bickel T., Anderson J., Obstacles to high-dimensional particle filtering, Monthly Weather Review Vol. 136, pp. 4629-4640, (2008).

18. DNV-RP-F101 Det Norske Veritas Recommended practice Corroded pipelines (2010).

19. Da Costa Mattos H.S., Reis J. M. L, Paim L. M., Silva da M. L., Lopes Junior R., Perrut V.A., Failure analysis of corroded pipelines reinforced with composite repair systems, Engineering Failure Analysis, 59, pp. 223-236, (2016).

20. Mazurkiewicz Ł., Tomaszewski M., Małachowski J., Sybilski K., Chebakov M., Witek M., Yukhymets P., Dmitrienko R., Experimental and numerical study of steel pipe with part-wall defect reinforced with fibre glass sleeve, International Journal of Pressure Vessels and Piping, Vol. 149, pp. 108-119, (2017).

21. Gerwatowski, D., Analiza czynników wpływających na przepustowość układu przesyłowego gazu ziemnego, PhD Thesis, Warsaw University of Technology (2011). 